Thorax (1968), 23, 350.

\title{
A rare type of atrial septal defect
}

\author{
R. J. M. M C C OR M A C K, D. P I C K ER IN G, A N D I I I S M I T H \\ From the Departments of Thoracic Surgery, Cardiology, and Pathology, University of Edinburgh
}

A previously unrecorded cardiac anomaly is described. A defect existed in the lowest part of the atrial septum straddling the orifice of the inferior vena cava so that this vessel opened into both atria. There was an associated anomalous connexion of the right pulmonary veins to the right atrium. The septal defect was recognized only at necropsy. As in the case of a sinus venosus atrial defect, it is suggested that the fundamental embryological error responsible for the production of the rare defect here described is laevo-position of the sino-atrial orifice.

There are only two cases recorded in the literature of drainage of the inferior vena cava into the left atrium (Gardner and Cole, 1955 ; Meadows, Bergstrand, and Sharp, 1961) and in both the atrial septum was intact. The purpose of this paper is to describe a previously unrecorded abnormality in which the inferior vena cava drained into both atria and there was an associated defect of the lowest part of the atrial septum.

\section{CASE REPORT}

A 6-year-old boy had been followed in the Paediatric Cardiology Clinic for four years. The mother reported that the boy sometimes became slightly blue on exertion: he was rather undersized but not recognizably disabled. In hospital cyanosis was never noted. A grade $4 / 6$ ejection systolic murmur was maximal in the pulmonary area and associated with a thrill. and there was a soft tricuspid diastolic murmur. An ejection click was not present: there was fixed splitting of the pulmonary second sound. The blood pressure was $100 / 60 \mathrm{~mm}$. $\mathrm{Hg}$. The chest radiograph was within normal limits and the electrocardiogram showed right ventricular hypertrophy. Cardiac catheterization in May 1965 demonstrated a systolic gradient of $50 \mathrm{~mm}$. $\mathrm{Hg}$ across the pulmonary valve and increased oxygenation of the right atrial blood. There was no increased oxygenation of the samples of caval blood. The catheter was passed into a right upper pulmonary vein and it was not certain whether the catheter entered this vein directly from the right atrium or by way of an atrial septal defect.

A diagnosis was made of pulmonary valvular stenosis associated with either an atrial septal defect or anomalous connexion of the right pulmonary veins to the right atrium. We deal with lesions of this type by open operation under hypothermia rather than using cardiopulmonary bypass; the child was therefore accepted for operation by this technique.
At operation on 4 November 1965 the pulmonary stenosis was relieved by an open transarterial valvulotomy. The findings inside the right atrium are shown in Figure 1. There was a small patent foramen ovale and the atrial septum appeared otherwise intact. Anomalous right pulmonary veins entered the right atrium directly and were transposed to the left atrium through a defect created along the line shown in Figure 1. The boy made a normal recovery from this operation, but a few days later he was noticed to be intermittently cyanosed at rest. It was thought that the cyanosis was attributable to the well-recognized error of inadvertent transposition of the inferior vena cava to the left atrium as a result of picking up the Eustachian valve at the time of closure of the atrial septal defect. This caval transposition was confirmed by injection of dye into the femoral vein and the appearance of the dye at the left ear after 2 seconds.

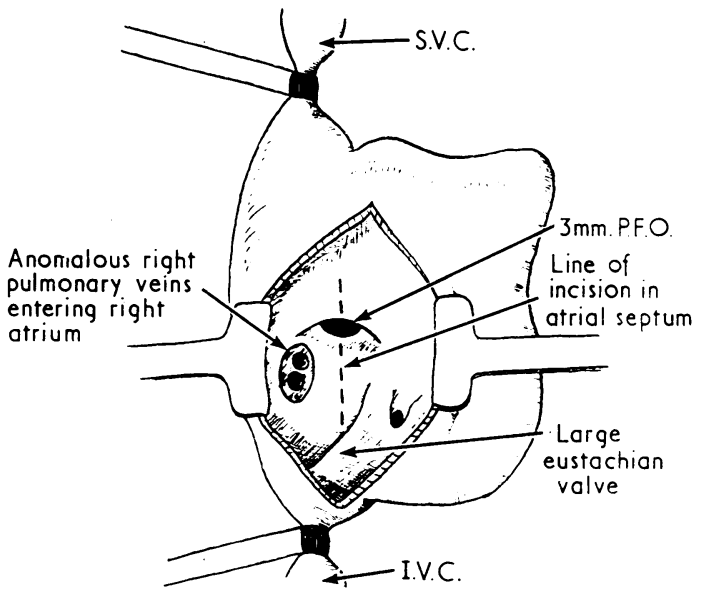

FIG. 1. Appearances found within right atrium at initial operation. 
A second operation was therefore carried out two weeks after the first, again under hypothermia. The transposition of the inferior vena cava was confirmed; the atrial defect was reopened and the septum was closed to the right of the right pulmonary venous orifices and to the left of a cannula placed in the inferior vena cava from the right atrium (Fig. 2). In the recovery room the patient had two cardiac arrests and on the second occasion attempts at resuscitation failed.

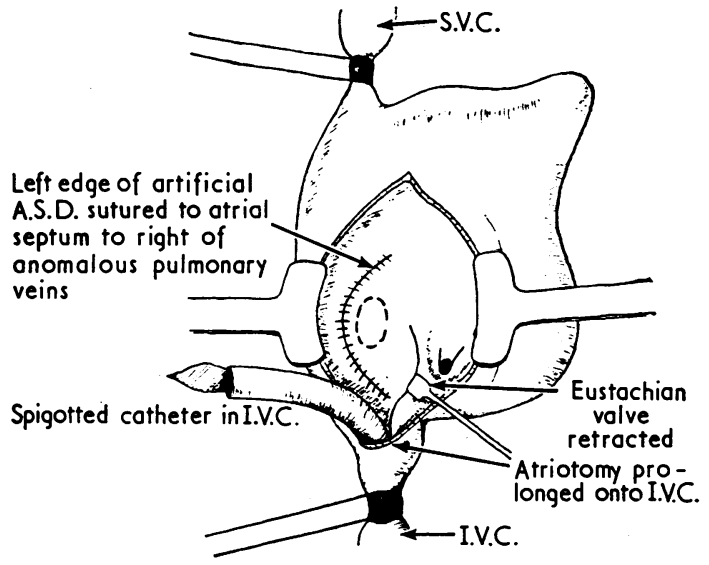

FIG. 2. Second operative procedure showing closure of atrial septum to right of anomalous veins and to left of a cannula placed in inferior vena cava.

POST-MORTEM FINDINGS At necropsy the important findings were limited to the heart. The previously recognized anomalies were confirmed and they all appeared to have been adequately corrected. There was an additional, unrecognized anomaly, namely a defect (Fig. 3) lying at the bottom of the atrial septum so that the lower edge of the septum straddled the orifice of the inferior vena cava, which communicated

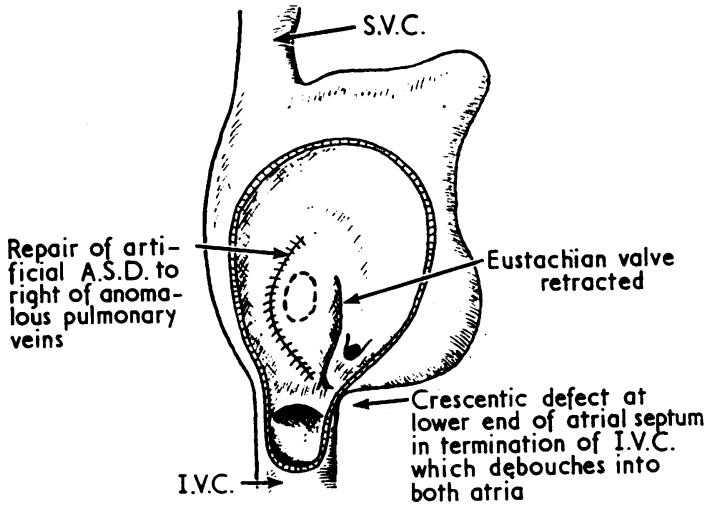

FIG. 3. Rare atrial defect at orifice of inferior vena cava as displayed at necropsy.

more with the left atrium than with the right. The defect was anatomically similar to the sinus venosus type of defect found at the top of the atrial septum. During open surgery the defect was completely hidden by the tape occluding the inferior vena cava and would also have been so under bypass. The coronary sinus was normal.

\section{EMBRYOLOGY}

The anatomical similarity between the defect described here and the well-known sinus venosus or high type of atrial septal defect occurring at the top of the atrial septum made the authors think that the two defects must have a similar embryological explanation. A description of the development of a sinus venosus defect has been given by Harley (1958). He believed that the error leading to the production of a sinus venosus defect was a failure of the sino-atrial orifice to shift as far to

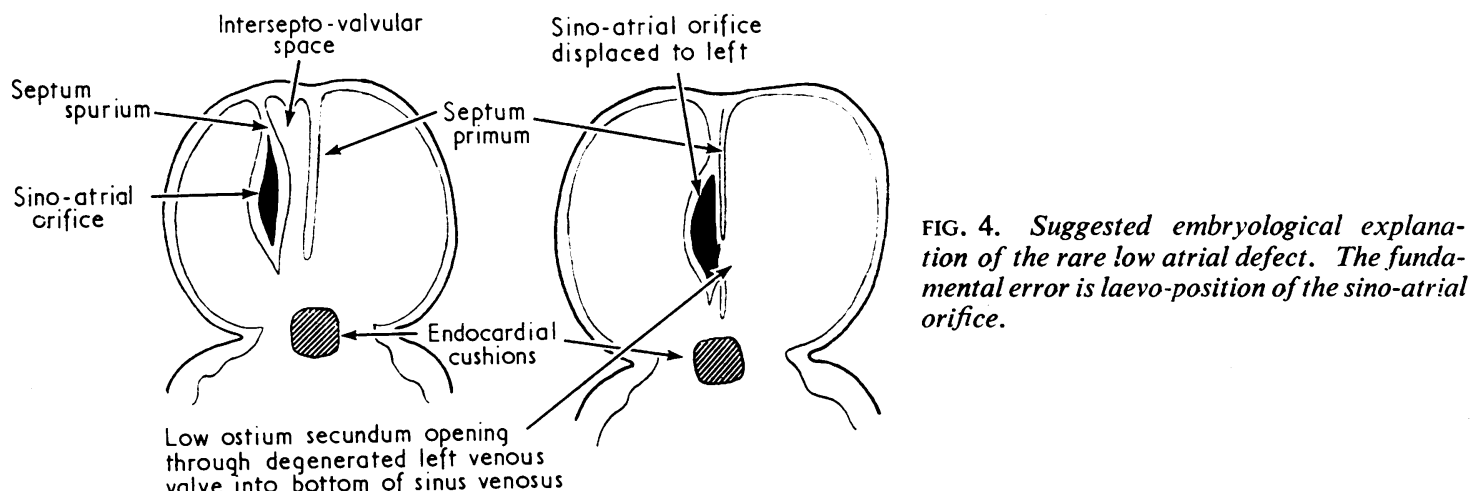


the right as it should. As a result (Fig. 4) the left venous valve developed in contact with the septum primum and there was no intersepto-valvular space. When the dorsicephalic part of the septum primum degenerated to form the ostium secundum, the contiguous left venous valve also regressed, with the resultant production of a defect in the upper part of the atrial septum between the left atrium on the one hand and the upper part of the sinus venosus on the other. As the sinus venosus was absorbed into the right atrium, the defect came to lie immediately below the orifice of the superior vena cava.

In the light of Harley's explanation, we postulate a similar mechanism to account for the development of the defect reported in this paper. We assume that the same error of laevo-position of the sino-atrial orifice exists. If the ostium secundum is formed low in the septum primum by degeneration of the latter's dorsicaudal instead $\stackrel{\vec{F}}{\stackrel{\vec{S}}{ }}$ of its dorsicephalic portion, then this would be 0 accompanied by regression of the inferior part of $\frac{}{\sigma}$ the left venous valve and the resultant defect $\frac{\bar{\omega}}{}$ would lie at the lower limit of the atrial $\mathbb{\varnothing}$ septum between the left atrium on the one hand and the lowest part of the sinus venosus on the other. With atrial absorption of the sinus venosus, the defect would come to lie immediately above $\overrightarrow{\vec{\omega}}$ the orifice of the inferior vena cava, in the position described in the case we here report.

\section{REFERENCES}

Gardner, D. L., and Cole, L. (1955). Long survival with inferior vena cava draining into left atrium. Brit. Heart J., 17, 93 .

Harley, H. R. S. (1958). The sinus venosus type of interatrial septal defect. Thorax, 13, 12.

Meadows, W. R., Bergstrand, I., and Sharp, J. T. (1961). Isolated anomalous connection of a great vein to the left atrium. The syndrome of cyanosis and clubbing, "normal" heart, and left $c$ ventricular hypertrophy on electrocardiogram. Circulation, 24, 